\title{
Promising loci of variable numbers of tandem repeats for typing Beijing family Mycobacterium tuberculosis
}

\author{
Yoshiro Murase, Satoshi Mitarai, Isamu Sugawara, Seiya Kato \\ and Shinji Maeda
}

Correspondence

Shinji Maeda

maeda@jata.or.jp

Received 8 August 2007

Accepted 13 March 2008

\author{
Research Institute of Tuberculosis, Japan Anti-Tuberculosis Association, 3-1-24 Matsuyama, \\ Kiyose, Tokyo 204-8533, Japan
}

\begin{abstract}
We analysed the genotypes of 325 Mycobacterium tuberculosis clinical isolates obtained during 2002 throughout Japan. The genotyping methods included insertion sequence IS6110 RFLP, spoligotyping and variable number of tandem repeat (VNTR) analyses. Clustered isolates revealed by IS6110 RFLP analysis accounted for $18.5 \%$ (60/325) of the isolates. Beijing genotype tuberculosis (TB) accounted for 73.8\% (240/325) of the isolates. Using VNTR, we analysed 35 loci, including 12 standard mycobacterial interspersed repetitive units and 4 exact tandem repeats. The discriminatory power of these 16 loci was low. Using VNTR analyses of the 35 loci, 12 loci (VNTRs 0424, 0960, 1955, 2074, 2163b, 2372, 2996, 3155, 3192, 3336, 4052 and 4156) were selected for the genotyping of Beijing genotype strains. Comparison of the discriminatory power of the 12-locus VNTR [Japan Anti-Tuberculosis Association (JATA)] to that of the 15-locus and 24-locus VNTRs proposed by Supply et al. (2006) showed that our established VNTR system was superior to the reported 15-locus VNTR and had almost equal discriminatory power to the 24-locus VNTR. This 12-locus VNTR (JATA) can therefore be used for TB genotyping in areas where Beijing family strains are dominant.
\end{abstract}

\section{INTRODUCTION}

RFLP analysis using insertion sequence IS6110 is the gold standard of tuberculosis (TB) genotyping (Cave et al., 1991; Kremer et al., 1999; van Embden et al., 1993). However, this RFLP analysis has many associated problems (Mostrom et al., 2002).

Spoligotyping has been used throughout the world as a convenient and reproducible PCR-based method for genotyping (Kamerbeek et al., 1997). Nevertheless, it provides little information related to Beijing family strains because spoligotypes of this genotype family show very little variation (Glynn et al., 2002; Kremer et al., 2004). In the countries of eastern Asia, the frequency of Beijing genotype TB is high. For that reason, spoligotyping is ineffective to discriminate unrelated isolates.

Analysis of variable number of tandem repeat (VNTR) loci is a promising PCR-based typing method. In VNTR analysis, some mini-satellite loci in the Mycobacterium tuberculosis genome are amplified using PCR; then their copy numbers are determined (Smittipat \& Palittapongarnpim, 2000;

Abbreviations: CDC, Centers for Disease Control and Prevention; ETR, exact tandem repeat; HGDI, Hunter-Gaston discriminatory index; JATA, Japan Anti-Tuberculosis Association; MIRU, mycobacterial interspersed repetitive unit; TB, tuberculosis; VNTR, variable number of tandem repeats.
Supply et al., 2000). In fact, VNTR analysis with the standard 12 loci of mycobacterial interspersed repetitive units (MIRUs) has been used in the USA and Europe (Blackwood et al., 2004; Mazars et al., 2001). The Centers for Disease Control and Prevention (CDC) in the USA have adopted the standard 12-locus MIRU-VNTR for TB analyses (Cowan et al., 2005; CDC, 2004). However, the discriminatory power of 12-locus MIRU-VNTR has been found to be insufficient (Supply et al., 2006), which similarly applies to Beijing genotype TB analyses (Kam et al., 2005; Kremer et al., 2005; Nikolayevskyy et al., 2006). Several reports have described other loci. Almost all corresponding loci have been described in the relevant literature (Iwamoto et al., 2007; Kam et al., 2006; Wada et al., 2007).

It is noteworthy that a new 15-locus or 24-locus MIRUVNTR method has been proposed in Europe (Oelemann et al., 2007; Supply et al., 2006). These new VNTR loci have had a distinct impact on genotyping of the Beijing family, but some large clusters remain (Iwamoto et al., 2007). Therefore, several VNTR loci must be added to the reported 15-locus VNTR method for analyses of Beijing genotype strains (Yokoyama et al., 2007).

For exploring the diversity of VNTR loci, we performed genotyping of IS6110 RFLP, spoligotyping and 35-locus VNTR analysis using TB isolates from all over Japan. Based 
on these results, we propose a VNTR system that has the same discriminatory power as RFLP for analysis of a minimum number of loci in countries where the Beijing family has spread. Moreover, the results provide useful information for molecular epidemiological analyses of TB in regions where the Beijing genotype is dominant.

\section{METHODS}

M. tuberculosis isolates. In all, $325 \mathrm{M}$. tuberculosis isolates, 3-10 strains per prefecture, were selected randomly from among 3122 isolates collected for a drug-resistance survey conducted in Japan in 2002 by the Tuberculosis Research Committee (RYOKEN). The isolates that we analysed had been originally considered as low cluster because the possibility of contact among patients was quite low. For comparing results of RFLP and VNTR analyses, $76 \mathrm{M}$. tuberculosis isolates were collected from 25 suspected epidemic outbreaks, along with epidemiological information. The epidemiologically linked 17 clusters (54 strains) showed the same RFLP pattern in each case. The 8 initially suspected TB patient clusters ( 22 strains) were found to be independent simultaneous occurrences: each strain had a different RFLP pattern. Mycobacterial genomic DNA was prepared from bacteria grown on Ogawa medium using an Isoplant kit (Nippon Gene).

Molecular typing methods. For this study, IS6110 RFLP typing was performed according to a standardized protocol (van Embden et al., 1993); band patterns were analysed using the BioNumerics software package (Applied Maths). In RFLP analysis, strains that have an identical band pattern were categorized as a cluster. Spoligotyping was also performed according to a standard protocol (Kamerbeek et al., 1997). Sequences of primers used for amplification of 12 MIRU loci (MIRU-02, 04, 10, 16, 20, 23, 24, 26, 27, 31, 39 and 40), 4 exact tandem repeats (ETRs) (ETR-A, B, C and F) and 19 other loci (VNTRs 0424, 1612, 1895, 1955, 2074, 2163a, 2163b, 2347, 2372, $2401,3155,3171,3232,3336,3690,3820,4052,4120$ and 4156) for VNTR were selected (Frothingham \& Meeker-O'Connell, 1998; Iwamoto et al., 2007; Roring et al., 2002; Smittipat et al., 2005; Supply et al., 2001, 2006). The VNTR typing was performed using Ex Taq with GC PCR buffer I (Takara Bio). The PCR mixture was prepared in a $20 \mu \mathrm{l}$ volume with $1 \times$ GC PCR buffer I, $0.5 \mathrm{U}$ Ex Taq, $200 \mu \mathrm{M}$ each of four dNTPs, $0.5 \mu \mathrm{M}$ each of the primer set and $10 \mathrm{ng}$ template DNA. Then PCR was carried out for all loci under the following conditions: initial denaturation at $94{ }^{\circ} \mathrm{C}$ for $5 \mathrm{~min}$, and then 35 cycles of $94{ }^{\circ} \mathrm{C}$ for $30 \mathrm{~s}, 63{ }^{\circ} \mathrm{C}$ for $30 \mathrm{~s}$ and $72{ }^{\circ} \mathrm{C}$ for $3 \mathrm{~min}$, followed by a final extension at $72{ }^{\circ} \mathrm{C}$ for $7 \mathrm{~min}$.

Estimation of molecular size of amplified DNA fragments. The sizes of the amplified DNA fragments were determined using a capillary array electrophoresis analysis system (i-Chip SV1210; Hitachi Electronics Engineering) (Sonehara et al., 2006) or an ABI 3130 genetic analyser (Applied Biosystems) with the GeneMapper program (Applied Biosystems). The $i$-Chip was used to calibrate VNTR analysis and the genetic analyser was used to analyse the standard 12 loci MIRU (Iwamoto et al., 2007; Supply et al., 2001). Alternatively, the PCR products were analysed in a $2-2.5 \%$ agarose gel. Their respective copy numbers were calculated from their size and assigned according to the number of repeats for each locus (Frothingham \& Meeker-O'Connell, 1998; Kremer et al., 2005; Skuce et al., 2002; Supply et al., 2001). The calculation accuracy was confirmed through analysis of M. tuberculosis H37Rv.

Allelic diversity and discrimination. The allelic diversity $(h)$ at each VNTR locus was calculated using the index $h=1-\Sigma x_{i}^{2}$, where $x_{i}$ is the frequency of the $i$ th allele at the locus, as used in other studies
(Kremer et al., 2005; Sun et al., 2004). The Hunter-Gaston discriminatory index (HGDI) was calculated in accordance with a method explained in another paper (Hunter \& Gaston, 1988) to evaluate the combination of some VNTR loci.

Combinations of VNTR loci. For the following combination of VNTR loci, the HGDI were compared: 15-locus VNTR [Supply (15)] - VNTRs 0424, 0577, 0580, 0802, 0960, 1644, 1955, 2163b, 2165, 2401, 2996, 3192, 3690, 4052 and 4156; 24-locus VNTR [Supply (24)] - Supply (15)+VNTRs 0154, 2059, 2347, 2461, 2531, 2687, 3007, 3171 and 4348; 12-locus VNTR [Japan Anti-Tuberculosis Association (JATA) (12)] - VNTRs 0424, 0960, 1955, 2074, 2163b, 2372, 2996, 3155, 3192, 3336, 4052 and 4156.

\section{RESULTS AND DISCUSSION}

\section{RFLP, spoligotyping and MIRU-VNTR analyses in Japan}

From throughout Japan, 325 collected isolates were analysed using IS6110 RFLP (Table 1). The percentage of clustered isolates was $18.5 \%(60 / 325)$. In spoligotyping, 293 (90\%) isolates formed clusters; the maximum cluster size was 228. The largest cluster was composed of the Beijing genotype [70.2\% (228/325)]. Because the number of Beijing-like strains was 12 (Kremer et al., 2004), the total of Beijing genotype strains used in this experiment was 240 $(73.8 \%)$. The Beijing family strains were confirmed to be dominant in Japan. The CDC in the USA has adopted the standard 12-locus MIRU-VNTR for TB analyses (Cowan et al., 2005; CDC, 2004). We analysed the 325 TB isolates using 12-locus MIRU-VNTR and 16-locus VNTR (12 standard MIRU and 4 ETRs). There were only 89 unique types in the 12-locus MIRU-VNTR. The percentage of clustered isolates decreased from $72.6 \%$ (12-MIRUVNTR) to $62.8 \%$ (16-locus VNTR) when the ETR loci were added to 12-locus MIRU analysis. Both rates of clustered isolates in VNTR analysis were higher than in RFLP (18.5\%). Furthermore, the HGDI showed that the discriminatory power of IS6110 RFLP was the highest of all analyses used for this study: spoligotyping, 12-locus MIRUVNTR and 16-locus VNTR.

An optimal 15-locus VNTR has been proposed by a consortium of European and American laboratories as a new worldwide standard method for discriminating TB genotypes using 52 different groups of related strains from different countries (Supply et al., 2006). This VNTR method can discriminate in greater detail than the standard 12-locus MIRU-VNTR when the Beijing genotype of TB is analysed. However, it has been reported that the discriminatory power of 15-locus VNTR is insufficient for analysis of the Beijing genotype (Iwamoto et al., 2007). The frequency of the Beijing family in Japan ( $80 \%$ of total TB) (Yokoyama et al., 2007) differs greatly from that in the USA (16\%) and European countries (4\%) (Filliol et al., 2002). For that reason, new loci are necessary to analyse the Beijing family more effectively in Japan, Korea, China and other Asian countries. 
Table 1. Comparison of the discriminatory power of IS6110 RFLP, spoligotyping and VNTR analyses

\begin{tabular}{|c|c|c|c|c|c|c|}
\hline Typing method & $\begin{array}{l}\text { Total no. of } \\
\text { type patterns }\end{array}$ & $\begin{array}{c}\text { No. of } \\
\text { unique types }\end{array}$ & $\begin{array}{l}\text { No. of } \\
\text { clusters }\end{array}$ & $\begin{array}{c}\text { No. of } \\
\text { clustered } \\
\text { isolates }(\%)\end{array}$ & $\begin{array}{c}\text { Maximum } \\
\text { no. of isolates } \\
\text { in a cluster }\end{array}$ & $\mathrm{HGDI}^{\star}$ \\
\hline Spoligotyping & 45 & 32 & 13 & $293(90.2)$ & 228 & 0.501 \\
\hline 12-locus MIRU-VNTR & 127 & 89 & 38 & $236(72.6)$ & 61 & 0.944 \\
\hline $\begin{array}{l}\text { 10-locus VNTR (8-locus VNTR + VNTRs } 2996 \text { and } \\
\text { 3155) }\end{array}$ & 297 & 276 & 21 & $49(15.1)$ & 4 & 0.999 \\
\hline $\begin{array}{l}\text { 12-locus VNTR (JATA) (10-locus VNTR + VNTRs } \\
0960 \text { and 3192) }\end{array}$ & 302 & 284 & 18 & $41(12.6)$ & 4 & 0.999 \\
\hline 15-locus VNTR (Supply) & 291 & 269 & 22 & $56(17.2)$ & 6 & 0.999 \\
\hline
\end{tabular}

${ }^{\star}$ HGDI was calculated as described in Methods.

\section{VNTR analyses of 19 loci}

Different loci from those used for the USA or European countries must be examined when VNTR analysis is adopted for TB genotyping in Japan. Actually, VNTR analyses of 48 loci using 21 Beijing isolates have been reported (Smittipat et al., 2005). Some loci that had high $h$ for Beijing genotyping strains and other loci, such as Queen's University Belfast (QUB), were selected (Skuce et al., 2002). In fact, 19 loci of $325 \mathrm{~TB}$ isolates were analysed using VNTR (Table 2). It was generally difficult to obtain the exact copy number using agarose gel electrophoresis in VNTR analysis when the molecular size of the PCR product was greater than $1 \mathrm{~kb}$. More than 15 copies of repetitive units (larger than $1 \mathrm{~kb}$ ) were detected at VNTRs $2163 \mathrm{a}, 2163 \mathrm{~b}, 1895,3232,3336,3820$ and 4120 . Only a few isolates had more than 15 copies of repetitive units at the loci of VNTRs 2163b, 1895 and 3336. However, for analyses of VNTRs 2163a, 3232, 3820 and 4120, more than $4 \%$ of total isolates $(18,86,50$ and 14 strains, respectively) had 15 or more copies. For that reason, it was difficult to interpret the exact copy number. Furthermore, multiple PCR products were detected at the respective loci of VNTRs 2163b, 3232, 3336, 3820, 4120 and 4156. These had the potential to be unstable loci in VNTR analysis, and might be in the process of copy number change. Moreover, no PCR product was found in analyses of VNTRs 2163a, 2163b, 4052, 0424 and 2347.

From Japan, it was reported that VNTRs 3232, 3820 and 4120 are the hyper-variable loci (Iwamoto et al., 2007; Wada et al., 2007; Yokoyama et al., 2007). More than $5 \%$ of total isolates presented analytical problems (absence of PCR product, PCR products difficult to interpret or amplification of multiple alleles) at the loci of VNTRs 2163a, 3232, 3820 and 4120. Therefore, to obtain stable and precise VNTR results, these four loci were excluded from VNTR analyses of Beijing family strains.

\section{New combination of VNTR loci for TB typing in Japan}

The discriminatory power of each locus, based on the $h$ values of all isolates, is presented in Table 3. The $h$ of nonBeijing family strains was higher than that of the Beijing strain, except for VNTRs 0424, 2163b and 4156. These three loci were indispensable for distinction of the Beijing family.

One purpose of this study is to establish a new VNTR system with equal discriminatory power to that of RFLP using a minimum loci analysis in countries where the Beijing family is prevalent. Combinations of some loci, such as the top 8 loci (8-locus VNTR), top 10 loci (10locus VNTR) or top 12 loci (12-locus VNTR), were compared to those of IS6110 RFLP (Table 1) in terms of several characteristics, such as the number of unique isolates and clusters. In fact 8-locus VNTR had resolution performance that was equal to IS6110 RFLP analysis in terms of the number of independent isolates and the rate of clustered isolates. Therefore, the 10-locus and 12-locus VNTRs were superior to IS6110 RFLP. However, the quantities of clusters in VNTR analysis were comparable to those of RFLP only when 12-locus VNTR was used. Consequently, the primer set of the 12-locus VNTR (JATA) was ultimately selected for VNTR analysis in Japan.

\section{Comparison of the discriminatory power of IS6110 RFLP and VNTR analyses}

A novel standard, optimized 15-locus and 24-locus VNTR primer sets (Supply et al., 2006), was proposed recently (Table 3). The respective discriminatory powers of IS6110 RFLP and VNTRs [15-locus (Supply) (Supply et al., 2006), 24-locus (Supply) and 12-locus (JATA)] were compared using the percentage of clustered isolates (Fig. 1). The fraction of clustered isolates of 12-locus VNTR (JATA) 
Table 2. The allelic profiles of 19 additional VNTR loci in $325 \mathrm{M}$. tuberculosis strains

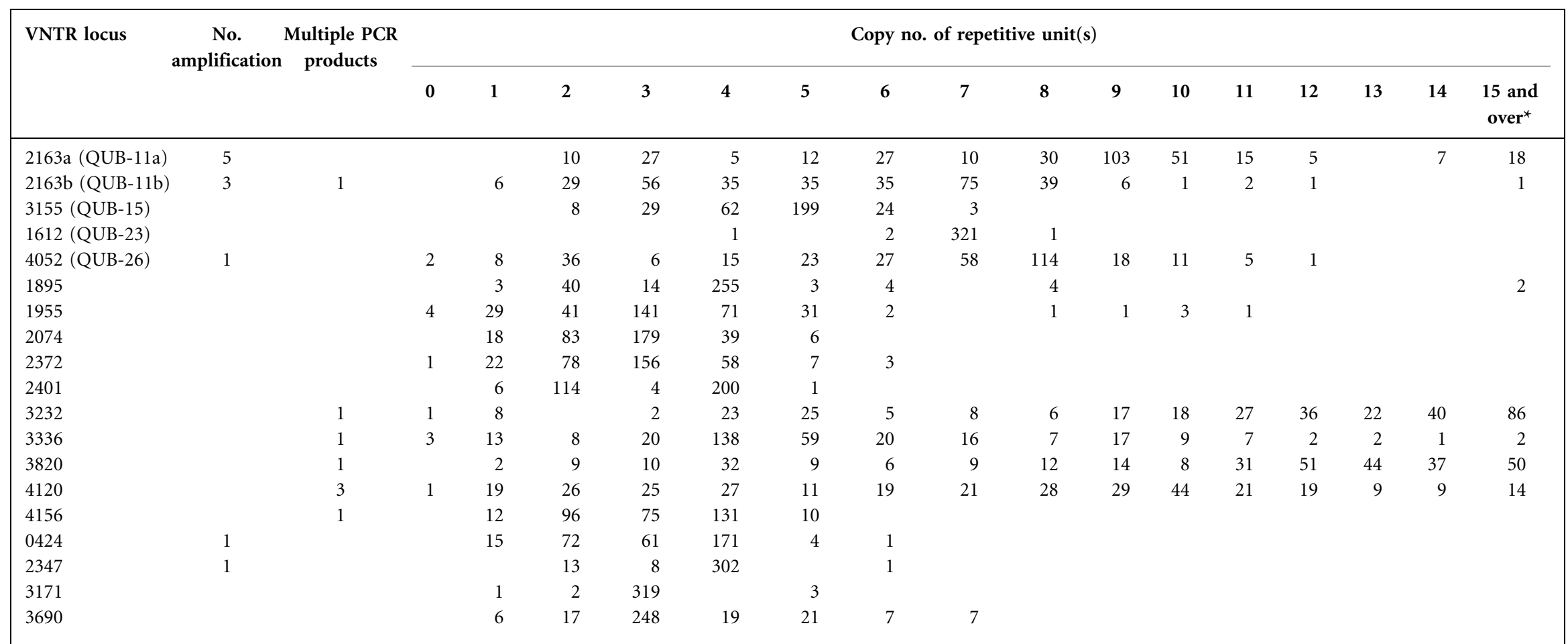


Table 3. The $h$ values for each locus and details of the VNTR loci selected for the different VNTR analyses

\begin{tabular}{|c|c|c|c|c|c|c|c|c|c|}
\hline \multirow[t]{2}{*}{ No. } & \multirow[t]{2}{*}{ Locus } & \multirow[t]{2}{*}{ Alias } & \multicolumn{3}{|c|}{$\boldsymbol{h}^{*}$} & \multirow{2}{*}{$\begin{array}{l}\text { This study } \\
12 \text { VNTR }\end{array}$} & \multicolumn{2}{|c|}{ Supply et al. (2006) $\dagger$} & \multirow{2}{*}{$\begin{array}{l}12 \text { MIRU } \\
+4 \text { ETRs }\end{array}$} \\
\hline & & & $\begin{array}{l}\text { All isolates } \\
\quad(n=325)\end{array}$ & $\begin{array}{l}\text { Beijing family } \\
\quad(n=240)\end{array}$ & $\begin{array}{l}\text { Non-Beijing } \\
\quad(n=85)\end{array}$ & & 24 VNTR & 15 VNTR & \\
\hline 1 & $2163 b$ & QUB $11 \mathrm{~b}$ & 0.855 & 0.815 & 0.802 & $x$ & $x$ & $x$ & \\
\hline 2 & 4052 & QUB 26 & 0.812 & 0.764 & 0.860 & $x$ & $x$ & $\times$ & \\
\hline 5 & 4156 & VNTR 4156 & 0.693 & 0.623 & 0.564 & $x$ & $x$ & $x$ & \\
\hline 6 & 2372 & VNTR 2372 & 0.675 & 0.595 & 0.672 & $x$ & & & \\
\hline 7 & 0424 & Mtub 04 & 0.635 & 0.468 & 0.425 & $x$ & $x$ & $x$ & \\
\hline 8 & 2074 & Mtub 24 & 0.614 & 0.591 & 0.615 & $x$ & & & \\
\hline 9 & 2996 & MIRU 26 & 0.591 & 0.314 & 0.667 & $x$ & $x$ & $x$ & $x$ \\
\hline 14 & 2165 & ETR A & 0.496 & 0.223 & 0.446 & & $x$ & $x$ & $x$ \\
\hline 15 & 0802 & MIRU 40 & 0.473 & 0.229 & 0.697 & & $\times$ & $x$ & $x$ \\
\hline 16 & 4348 & MIRU 39 & 0.472 & 0.156 & 0.543 & & $\times$ & & $\times$ \\
\hline 17 & 3690 & Mtub 39 & 0.406 & 0.215 & 0.745 & & $\times$ & $x$ & \\
\hline 18 & 1895 & VNTR 1895 & 0.367 & 0.337 & 0.447 & & & & \\
\hline 19 & 3239 & ETR F & 0.358 & 0.237 & 0.575 & & & & $x$ \\
\hline 20 & 1644 & MIRU 16 & 0.345 & 0.258 & 0.502 & & $x$ & $x$ & $x$ \\
\hline 21 & 2531 & MIRU 23 & 0.336 & 0.158 & 0.555 & & $\times$ & & $x$ \\
\hline 22 & 0580 & MIRU 4 & 0.189 & 0.049 & 0.484 & & $x$ & $x$ & $x$ \\
\hline 23 & 2461 & ETR B & 0.151 & 0.017 & 0.447 & & $\times$ & & $x$ \\
\hline 24 & 0577 & ETR C & 0.134 & 0.057 & 0.317 & & $\times$ & $x$ & $\times$ \\
\hline 33 (excluded) & 4120 & VNTR 4120 & 0.924 & 0.902 & 0.767 & & & & \\
\hline 34 (excluded) & 3820 & VNTR 3820 & 0.911 & 0.871 & 0.824 & & & & \\
\hline 35 (excluded) & $2163 a$ & QUB 11a & 0.836 & 0.752 & 0.858 & & & & \\
\hline
\end{tabular}

${ }^{\star} h$ represents the allelic diversity of each locus, calculated as described in Methods.

$\dagger$ The combination of 15- and 24-locus VNTR analyses were reported by Supply et al. (2006).

$(12.6 \%)$ was superior to that of RFLP $(18.5 \%)$ and $15-$ locus VNTR (Supply) (17.2\%) in this experiment. The respective discriminatory capabilities of 15-locus and 24locus VNTR (Supply) and 12-locus VNTR (JATA) were better than that of IS6110 RFLP.

Our newly established 12-locus VNTR (JATA) typing method had almost equivalent discriminatory power to that of 24-locus VNTR (Supply) for TB genotyping, although it uses only 12 loci. In the 12-locus VNTR (JATA), 8 loci overlap with the 15-locus and 24-locus VNTRs (Supply): 4 loci (VNTRs 2074, 2372, 3155 and 3336) are independent. In the Supply VNTR typing method (Supply et al., 2006), VNTR 3336 was discarded from the final selection for detection because of the following observations: it gave multiple PCR products, it did not give a PCR product and there was a lack of reproducibility between results obtained in independent laboratories. 'No PCR amplification' represents a characteristic typing datum; it indicates that the strain has different sequences in the primer binding site or has no such region. In addition, detection of a multiple PCR product might signify that a conversion of copy number is occurring at the locus, meaning that the genetic stability of such loci is not high. 


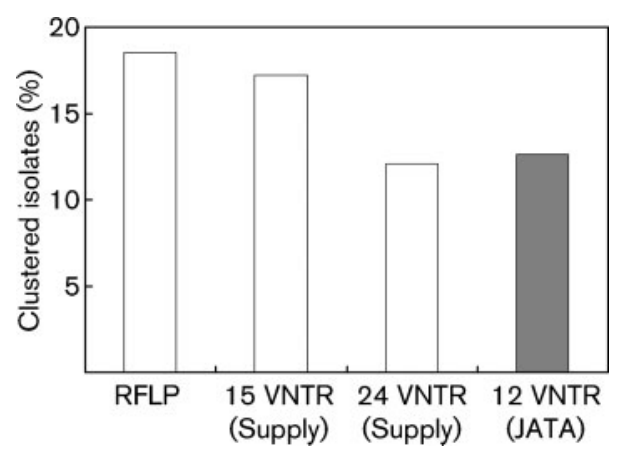

Fig. 1. Comparison of clustering rates in RFLP and VNTR analyses. The percentage of clustered isolates was calculated for each case.

Our data showed that the $h$ of VNTR 3336 gave a maximum value in non-Beijing TB analysis (Table 3 ). The genetic clock of VNTR 3336 in non-Beijing genotypes might be faster than that of Beijing strains. Therefore, the locus of VNTR 3336 in non-Beijing strains might be unstable and that in Beijing strains might be stable. Consequently, the locus of VNTR 3336 might be used in 12-locus VNTR (JATA).

In this study, 325 independent isolates (with no epidemiological link) were collected and analysed. Results show that only four strains formed clusters in both the RFLP (60 strains) and VNTR (41 strains) methods: RKN 246, RKN 275, RKN 283 and RKN 319. The remaining clusters were subdivided using the other method. Cluster 08 of RFLP analysis comprised six strains including the four clustered strains. By 12-locus (JATA) VNTR, cluster 08 was further divided into three groups (Fig. 2). The two remaining strains (RKN 177 and RKN 215) showed independent patterns. Differences of copy number in the two strains were found in two loci. The respective discriminatory powers of RFLP and VNTR analyses were different. The maximum cluster sizes in IS6110 RFLP and JATA (12) VNTR were, respectively, 8 and 4 (data not shown). The 12-locus (JATA) VNTR had a higher discrimination power than RFLP analysis did.

\section{Analysis of clinical isolates from a suspected outbreak using 12-locus VNTR (JATA)}

For examining the effectiveness of the 12-locus VNTR (JATA), 25 clusters (76 strains in total) were analysed. Of them, 17 clusters (54 strains) for which the RFLP patterns were identical in each group had the same VNTR profile (data not shown). Eight initially suspected TB patient clusters (22 strains) were not outbreak related (concurrence of TB): their IS6110 RFLP showed different patterns. These strains are clearly independent. Therefore, the VNTR profile was also expected to show variant patterns in the respective groups. The VNTR analyses using 12-locus VNTR (JATA) showed that each strain had a different profile in at least 5 loci, except for case 6 . Analyses of the other isolates also revealed that the discriminatory power of 12-locus VNTR (JATA) was roughly equivalent to that of IS6110 RFLP analysis.

In case six, strains S-054 and S-160 differed by only one locus (VNTR 2074). Their RFLP patterns were notably different (data not shown). This concomitant change in two independent markers suggests that these two isolates originated from independent clones. They could still very well represent an outbreak if strains were to have highly similar RFLP (differing by only one or two bands) and have identical VNTR profiles. It is impossible to determine whether or not this was a mass infection case. For that reason, a conclusion should be offered only after considering all results, including contact examination and epidemiological investigation.

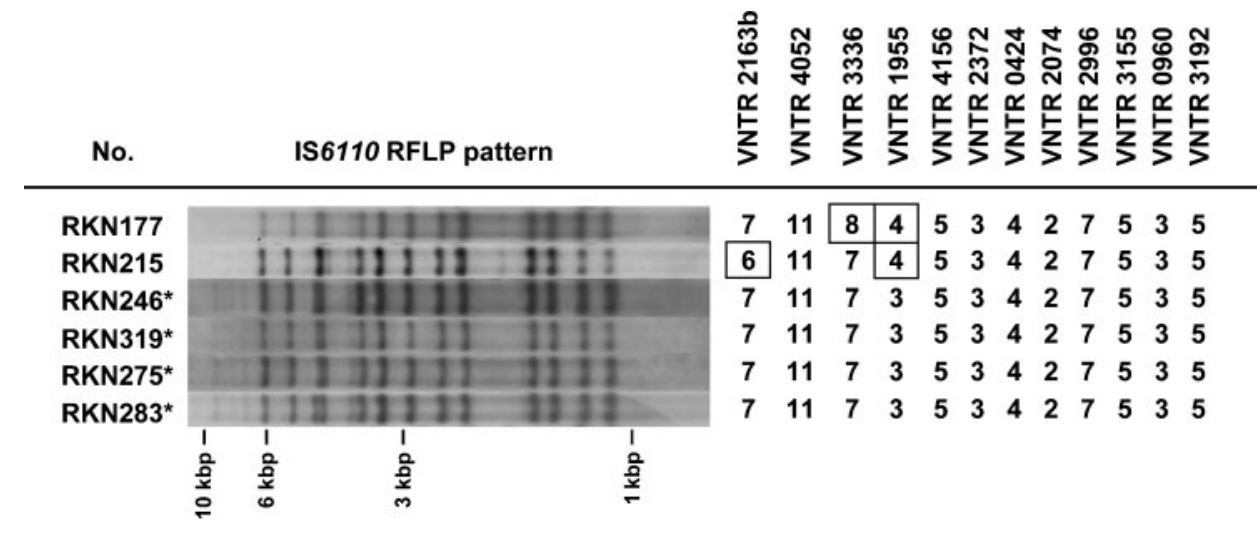

Fig. 2. The subdivision of cluster 08 in IS6110 RFLP using 12-locus (JATA) VNTR. Cluster 08 in RFLP analysis comprised six strains. The JATA-VNTR divided cluster 08 into three groups. The isolates marked with an asterisk (RKN246, RKN275, RKN283 and RKN319) were clustered using both RFLP and VNTR analyses. Differences in VNTR patterns are boxed. 
In VNTR analysis, the frequency of copy number conversion at each locus remains unclear. The copy number was found to be changed at VNTRs 3232 and 3690 (Iwamoto et al., 2007) when the cloned isolate was cultured for 7-23 months. Furthermore, one repeat difference has been reported at MIRU-26 (Savine et al., 2002). In these cases, conversion of the copy number was found at a single locus. Other reports have described that the copy quantities of VNTR changed at multiple loci (Supply et al., 2006; van Deutekom et al., 2005). The frequency of conversion of tandem repeats depended on each locus. Therefore, the accumulation of data related to the stability of each locus is a task of pressing urgency.

The TB isolates without epidemiological links were clearly classified according to differences of VNTR profiles (Oelemann et al., 2007). It is impossible to conclude whether two strains are derived from the same origin, or not, merely by VNTR analysis when a difference of copy number is observed in one or two loci. When more detailed typing is required in a survey IS6110 RFLP or other loci, including hyper-variable regions in the VNTR, might be used.

Molecular epidemiological analyses are useful both for transmission surveys and population-based retrospective studies. A recent report described that up to $14 \%$ of the strain clusters identified by IS6110 RFLP analyses might include false clustering (van Deutekom et al., 2005). Apparently, true TB genotyping clusters are obtainable through VNTR analysis because it can analyse $M$. tuberculosis objectively when suitable loci are selected.

For the present PCR platform, analyses of 8 or 12 samples are convenient. Herein, we propose this 12-locus VNTR as a useful means for TB genotyping where Beijing strains are prevalent. This is one report proposing new VNTR loci for Beijing strains. These loci do not represent a final decision. Additional loci can be chosen for TB genotyping in VNTR analysis if further discrimination is necessary. In addition, when the four loci selected here (VNTRs 2074, 2372, 3155 and 3336) are added to 15-locus VNTR (Supply), both genotypes of Beijing and non-Beijing can be analysed.

In this report, we propose new combinations of loci for VNTR analysis for the Beijing family. Furthermore, this study shows that JATA (12) VNTR has higher discriminatory power than IS6110 RFLP. However, the stability of each locus in VNTR analysis remains unknown. To obtain accumulated data, additional experiments are necessary.

\section{ACKNOWLEDGEMENTS}

The authors sincerely thank the members of the Tuberculosis Research Committee (RYOKEN) for permission to use their TB isolates for this study. This work was supported by grants from the Ministry of Health, Labour and Welfare (Research on Emerging and Re-emerging Infectious Diseases, Health Sciences Research Grants), the United States - Japan Cooperative Medical Science Program against Tuberculosis and Leprosy, and the Oyama Health Foundation.

\section{REFERENCES}

Blackwood, K. S., Wolfe, J. N. \& Kabani, A. M. (2004). Application of mycobacterial interspersed repetitive unit typing to Manitoba tuberculosis cases: can restriction fragment length polymorphism be forgotten? J Clin Microbiol 42, 5001-5006.

Cave, M. D., Eisenach, K. D., McDermott, P. F., Bates, J. H. \& Crawford, J. T. (1991). IS6110: conservation of sequence in the Mycobacterium tuberculosis complex and its utilization in DNA fingerprinting. Mol Cell Probes 5, 73-80.

CDC (2004). Guide to the Application of Genotyping to Tuberculosis Prevention and Control. Handbook for TB Controllers, Epidemiologists, Laboratorians, and Other Program Staff - June 2004. Atlanta, GA; Centers for Disease Control and Prevention. http://www.cdc.gov/tb/ genotyping/manual.htm.

Cowan, L. S., Diem, L., Monson, T., Wand, P., Temporado, D., Oemig, T. V. \& Crawford, J. T. (2005). Evaluation of a two-step approach for large-scale, prospective genotyping of Mycobacterium tuberculosis isolates in the United States. J Clin Microbiol 43, 688-695.

Filliol, I., Driscoll, J. R., Van Soolingen, D., Kreiswirth, B. N., Kremer, K., Valétudie, G., Anh, D. D., Barlow, R., Banerjee, D. \& other authors (2002). Global distribution of Mycobacterium tuberculosis spoligotypes. Emerg Infect Dis 8, 1347-1349.

Frothingham, R. \& Meeker-O'Connell, W. A. (1998). Genetic diversity in the Mycobacterium tuberculosis complex based on variable numbers of tandem DNA repeats. Microbiology 144, 1189-1196.

Glynn, J. R., Whiteley, J., Bifani, P. J., Kremer, K. \& van Soolingen, D. (2002). Worldwide occurrence of Beijing/W strains of Mycobacterium tuberculosis: a systematic review. Emerg Infect Dis 8, 843-849.

Hunter, P. R. \& Gaston, M. A. (1988). Numerical index of the discriminatory ability of typing systems: an application of Simpson's index of diversity. J Clin Microbiol 26, 2465-2466.

Iwamoto, T., Yoshida, S., Suzuki, K., Tomita, M., Fujiyama, R., Tanaka, N., Kawakami, Y. \& Ito, M. (2007). Hypervariable loci that enhance the discriminatory ability of newly proposed 15-loci and 24loci variable-number tandem repeat typing method on Mycobacterium tuberculosis strains predominated by the Beijing family. FEMS Microbiol Lett 270, 67-74.

Kam, K. M., Yip, C. W., Tse, L. W., Wong, K. L., Lam, T. K., Kremer, K., Au, B. K. \& van Soolingen, D. (2005). Utility of mycobacterial interspersed repetitive unit typing for differentiating multidrugresistant Mycobacterium tuberculosis isolates of the Beijing family. J Clin Microbiol 43, 306-313.

Kam, K. M., Yip, C. W., Tse, L. W., Leung, K. L., Wong, K. L., Ko, W. M. \& Wong, W. S. (2006). Optimization of variable number tandem repeat typing set for differentiating Mycobacterium tuberculosis strains in the Beijing family. FEMS Microbiol Lett 256, 258-265.

Kamerbeek, J., Schouls, L., Kolk, A., van Agterveld, M., van Soolingen, D., Kuijper, S., Bunschoten, A., Molhuizen, H., Shaw, R. \& other authors (1997). Simultaneous detection and strain differentiation of Mycobacterium tuberculosis for diagnosis and epidemiology. J Clin Microbiol 35, 907-914.

Kremer, K., van Soolingen, D., Frothingham, R., Haas, W. H., Hermans, P. W., Martín, C., Palittapongarnpim, P., Plikaytis, B. B., Riley, L. W. \& other authors (1999). Comparison of methods based on different molecular epidemiological markers for typing of Mycobacterium tuberculosis complex strains: interlaboratory study of discriminatory power and reproducibility. J Clin Microbiol 37, 26072618.

Kremer, K., Glynn, J. R., Lillebaek, T., Niemann, S., Kurepina, N. E., Kreiswirth, B. N., Bifani, P. J. \& van Soolingen, D. (2004). Definition 
of the Beijing/W lineage of Mycobacterium tuberculosis on the basis of genetic markers. J Clin Microbiol 42, 4040-4049.

Kremer, K., Au, B. K., Yip, P. C., Skuce, R., Supply, P., Kam, K. M. \& van Soolingen, D. (2005). Use of variable-number tandem-repeat typing to differentiate Mycobacterium tuberculosis Beijing family isolates from Hong Kong and comparison with IS6110 restriction fragment length polymorphism typing and spoligotyping. J Clin Microbiol 43, 314-320.

Mazars, E., Lesjean, S., Banuls, A. L., Gilbert, M., Vincent, V., Gicquel, B., Tibayrenc, M., Locht, C. \& Supply, P. (2001). High-resolution minisatellite-based typing as a portable approach to global analysis of Mycobacterium tuberculosis molecular epidemiology. Proc Natl Acad Sci U S A 98, 1901-1906.

Mostrom, P., Gordon, M., Sola, C., Ridell, M. \& Rastogi, N. (2002). Methods used in the molecular epidemiology of tuberculosis. Clin Microbiol Infect 8, 694-704.

Nikolayevskyy, V., Gopaul, K., Balabanova, Y., Brown, T., Fedorin, I. \& Drobniewski, F. (2006). Differentiation of tuberculosis strains in a population with mainly Beijing-family strains. Emerg Infect Dis 12, 1406-1413.

Oelemann, M. C., Diel, R., Vatin, V., Haas, W., Rusch-Gerdes, S., Locht, C., Niemann, S. \& Supply, P. (2007). Assessment of an optimized mycobacterial interspersed repetitive- unit-variable-number tandem-repeat typing system combined with spoligotyping for population-based molecular epidemiology studies of tuberculosis. J Clin Microbiol 45, 691-697.

Roring, S., Scott, A., Brittain, D., Walker, l., Hewinson, G., Neill, S. \& Skuce, R. (2002). Development of variable-number tandem repeat typing of Mycobacterium bovis: comparison of results with those obtained by using existing exact tandem repeats and spoligotyping. J Clin Microbiol 40, 2126-2133.

Savine, E., Warren, R. M., van der Spuy, G. D., Beyers, N., van Helden, P. D., Locht, C. \& Supply, P. (2002). Stability of variablenumber tandem repeats of mycobacterial interspersed repetitive units from 12 loci in serial isolates of Mycobacterium tuberculosis. J Clin Microbiol 40, 4561-4566.

Skuce, R. A., McCorry, T. P., McCarroll, J. F., Roring, S. M., Scott, A. N., Brittain, D., Hughes, S. L., Hewinson, R. G. \& Neill, S. D. (2002). Discrimination of Mycobacterium tuberculosis complex bacteria using novel VNTR-PCR targets. Microbiology 148, 519-528.

Smittipat, N. \& Palittapongarnpim, P. (2000). Identification of possible loci of variable number of tandem repeats in Mycobacterium tuberculosis. Tuber Lung Dis 80, 69-74.

Smittipat, N., Billamas, P., Palittapongarnpim, M., Thong-On, A., Temu, M. M., Thanakijcharoen, P., Karnkawinpong, O. \&
Palittapongarnpim, P. (2005). Polymorphism of variable-number tandem repeats at multiple loci in Mycobacterium tuberculosis. J Clin Microbiol 43, 5034-5043.

Sonehara, T., Kawazoe, H., Sakai, T., Ozawa, S., Anazawa, T. \& Irie, T. (2006). Ultra-slim laminated capillary array for high-speed DNA separation. Electrophoresis 27, 2910-2916.

Sun, Y. J., Bellamy, R., Lee, A. S., Ng, S. T., Ravindran, S., Wong, S. Y., Locht, C., Supply, P. \& Paton, N. I. (2004). Use of mycobacterial interspersed repetitive unit-variable-number tandem repeat typing to examine genetic diversity of Mycobacterium tuberculosis in Singapore. J Clin Microbiol 42, 1986-1993.

Supply, P., Mazars, E., Lesjean, S., Vincent, V., Gicquel, B. \& Locht, C. (2000). Variable human minisatellite-like regions in the Mycobacterium tuberculosis genome. Mol Microbiol 36, 762-771.

Supply, P., Lesjean, S., Savine, E., Kremer, K., van Soolingen, D. \& Locht, C. (2001). Automated high-throughput genotyping for study of global epidemiology of Mycobacterium tuberculosis based on mycobacterial interspersed repetitive units. J Clin Microbiol 39, 3563-3571.

Supply, P., Allix, C., Lesjean, S., Cardoso-Oelemann, M., Rüsch-Gerdes, S., Willery, E., Savine, E., de Haas, P., van Deutekom, H. \& other authors (2006). Proposal for standardization of optimized mycobacterial interspersed repetitive unit-variablenumber tandem repeat typing of Mycobacterium tuberculosis. J Clin Microbiol 44, 4498-4510.

van Deutekom, H., Supply, P., de Haas, P. E., Willery, E., Hoijng, S. P., Locht, C., Coutinho, R. A. \& van Soolingen, D. (2005). Molecular typing of Mycobacterium tuberculosis by mycobacterial interspersed repetitive unit-variable-number tandem repeat analysis, a more accurate method for identifying epidemiological links between patients with tuberculosis. J Clin Microbiol 43, 4473-4479.

van Embden, J. D., Cave, M. D., Crawford, J. T., Dale, J. W., Eisenach, K. D., Gicquel, B., Hermans, P., Martin, C., McAdam, R. \& other authors (1993). Strain identification of Mycobacterium tuberculosis by DNA fingerprinting: recommendations for a standardized methodology. J Clin Microbiol 31, 406-409.

Wada, T., Maeda, S., Hase, A. \& Kobayashi, K. (2007). Evaluation of variable numbers of tandem repeat as molecular epidemiological markers of Mycobacterium tuberculosis in Japan. J Med Microbiol 56, 1052-1057.

Yokoyama, E., Kishida, K., Uchimura, M. \& Ichinohe, S. (2007). Improved differentiation of Mycobacterium tuberculosis strains, including many Beijing genotype strains, using a new combination of variable number of tandem repeats loci. Infect Genet Evol 7, 499508. 